TABLE S1. Complete Air Quality Standard Comparison

\begin{tabular}{|c|c|c|c|c|c|c|c|c|c|c|c|c|c|c|c|c|c|c|c|c|c|c|c|c|c|c|c|c|c|c|c|}
\hline $\begin{array}{l}\text { Air } \\
\text { Pollutant }\end{array}$ & Time & D. & 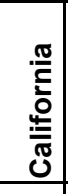 & $\begin{array}{l}0 \\
\stackrel{1}{1} \\
\end{array}$ & 己े & 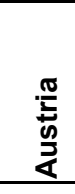 & 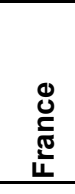 & 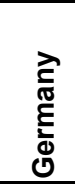 & 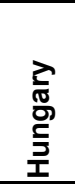 & $\begin{array}{l}\frac{n}{0} \\
\frac{0}{\pi} \\
\frac{\pi}{2} \\
\frac{0}{0} \\
\frac{0}{2} \\
\end{array}$ & 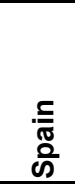 & 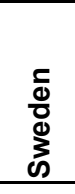 & |' & 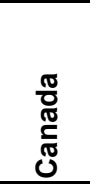 & $\begin{array}{l}\frac{\pi}{5} \\
\frac{0}{0} \\
\frac{0}{\alpha} \\
\end{array}$ & $\begin{array}{l}\dot{j} \\
\dot{m}\end{array}$ & $\begin{array}{l}\frac{0}{2 \pi} \\
\text { ț } \\
0\end{array}$ & $\begin{array}{l}0 \\
0 \\
0 \\
\\
0 \\
0\end{array}$ & 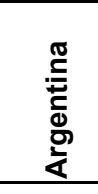 & 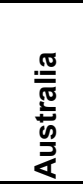 & $\begin{array}{l}\overline{\bar{N}} \\
\overline{\tilde{N}} \\
\end{array}$ & 竞 & 蒡 & $\begin{array}{l}\stackrel{0}{\bar{z}} \\
\underline{\underline{\underline{n}}}\end{array}$ & $\begin{array}{l}\frac{5}{\pi} \\
\frac{2}{\pi} \\
\frac{1}{2} \\
\end{array}$ & 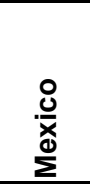 & $\begin{array}{l}N \\
z \\
\end{array}$ & 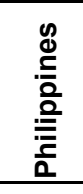 & $\begin{array}{l}\dot{x} \\
\dot{0} \\
\ddot{x} \\
\end{array}$ & 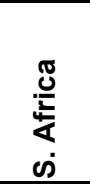 & $\begin{array}{c}\frac{D}{5} \\
\frac{\bar{\sigma}}{\bar{T}} \\
\frac{\bar{\sigma}}{F} \\
\end{array}$ \\
\hline $\begin{array}{l}\mathrm{CO} \\
{\left[\mathrm{mg} / \mathrm{m}^{3}\right]}\end{array}$ & $\begin{array}{l}1 \mathrm{hr} \\
8 \mathrm{hr} \\
24 \mathrm{hr} \\
\text { Annual }\end{array}$ & $\begin{array}{l}40 \\
10\end{array}$ & $\begin{array}{l}23 \\
10\end{array}$ & $\begin{array}{l}30 \\
10\end{array}$ & 10 & 10 & 10 & 10 & $\begin{array}{l}- \\
- \\
5 \\
2\end{array}$ & $\begin{array}{l}- \\
- \\
-\end{array}$ & $\begin{array}{r}- \\
10 \\
-\end{array}$ & $\begin{array}{r}- \\
10 \\
- \\
-\end{array}$ & 10 & $\begin{array}{r}35 \\
15 \\
- \\
-\end{array}$ & $\begin{array}{r}15 \\
6 \\
- \\
-\end{array}$ & $\begin{array}{r}28 \\
11 \\
- \\
-\end{array}$ & $\begin{array}{r}36.2 \\
15.7 \\
-\end{array}$ & $\begin{array}{l}34 \\
15\end{array}$ & $\begin{array}{r}57 \\
11 \\
- \\
-\end{array}$ & $\begin{array}{r}- \\
10 \\
- \\
-\end{array}$ & $\begin{array}{r}40 \\
10 \\
-\end{array}$ & $\begin{array}{r}10 \\
- \\
4 \\
-\end{array}$ & $\begin{array}{r}30 \\
10 \\
- \\
-\end{array}$ & $\begin{array}{l}4 \\
2 \\
- \\
-\end{array}$ & $\begin{array}{r}23 \\
11 \\
-\end{array}$ & $\begin{array}{r}- \\
12 \\
- \\
-\end{array}$ & $\begin{array}{r}30 \\
10 \\
- \\
-\end{array}$ & $\begin{array}{r}35 \\
10 \\
- \\
-\end{array}$ & $\begin{array}{r}29 \\
10 \\
- \\
-\end{array}$ & $\begin{array}{l}- \\
- \\
-\end{array}$ & $10 \mid$ \\
\hline $\begin{array}{l}\mathrm{NO}_{2} \\
{\left[\mu \mathrm{g} / \mathrm{m}^{3}\right]}\end{array}$ & $\begin{array}{l}1 \mathrm{hr} \\
24 \mathrm{hr} \\
\text { Annual }\end{array}$ & $\begin{array}{r}- \\
100\end{array}$ & 470 & $\begin{array}{r}200 \\
40\end{array}$ & $\begin{array}{r}200 \\
40\end{array}$ & $\begin{array}{r}200 \\
80 \\
30\end{array}$ & $\begin{array}{r}200 \\
- \\
40\end{array}$ & $\begin{array}{r}200 \\
- \\
40\end{array}$ & $\begin{array}{l}- \\
85 \\
70\end{array}$ & $\begin{array}{r}- \\
- \\
40\end{array}$ & $\begin{array}{r}200 \\
- \\
40\end{array}$ & $\begin{array}{l}90 \\
60 \\
40\end{array}$ & $\begin{array}{r}200 \\
40\end{array}$ & $\begin{array}{l}400 \\
200 \\
100\end{array}$ & $\begin{array}{r}400 \\
200 \\
60\end{array}$ & $\begin{array}{l}- \\
-\end{array}$ & $\begin{array}{r}400 \\
200 \\
-\end{array}$ & $\begin{array}{l}414 \\
207 \\
103\end{array}$ & $\begin{array}{r}850 \\
- \\
-\end{array}$ & $\begin{array}{r}226 \\
- \\
57\end{array}$ & $\begin{array}{r}320 \\
- \\
100\end{array}$ & $\begin{array}{r}120 \\
80 \\
40\end{array}$ & $\begin{array}{r}400 \\
150 \\
-\end{array}$ & $\begin{array}{l}- \\
80 \\
60\end{array}$ & $\begin{array}{r}- \\
75-113 \\
-\end{array}$ & $\begin{array}{r}396 \\
- \\
-\end{array}$ & $\begin{array}{r}200 \\
100 \\
-\end{array}$ & $\begin{array}{r}- \\
150 \\
-\end{array}$ & $\begin{array}{r}283 \\
151 \\
94\end{array}$ & $\begin{array}{r}377 \\
189 \\
94\end{array}$ & 32 \\
\hline $\begin{array}{l}\text { Ozone } \\
{\left[\mu \mathrm{g} / \mathrm{m}^{3}\right]}\end{array}$ & $\begin{array}{l}1 \mathrm{hr} \\
8 \mathrm{hr} \\
24 \mathrm{hr} \\
\text { Annual }\end{array}$ & $\begin{array}{r}235 \\
157 \\
- \\
-\end{array}$ & 180 & 120 & $\begin{array}{r}- \\
120 \\
- \\
-\end{array}$ & $\begin{array}{r}- \\
110 \\
- \\
-\end{array}$ & $\begin{array}{r}- \\
110 \\
- \\
-\end{array}$ & $\begin{array}{r}- \\
110 \\
- \\
-\end{array}$ & $\begin{array}{r}- \\
- \\
100 \\
-\end{array}$ & $\begin{array}{r}- \\
120 \\
- \\
-\end{array}$ & $\begin{array}{r}- \\
120 \\
- \\
-\end{array}$ & $\begin{array}{r}- \\
110 \\
- \\
-\end{array}$ & 120 & $\begin{array}{r}160 \\
127 \\
50 \\
30\end{array}$ & $\begin{array}{r}160 \\
- \\
50 \\
-\end{array}$ & $\begin{array}{l}- \\
- \\
-\end{array}$ & $\begin{array}{r}165 \\
- \\
- \\
-\end{array}$ & 157 & $\begin{array}{r}196 \\
- \\
- \\
-\end{array}$ & $\begin{array}{r}196 \\
157^{*} \\
- \\
-\end{array}$ & $\begin{array}{r}160 \\
- \\
- \\
-\end{array}$ & $\begin{array}{r}160 \\
- \\
- \\
-\end{array}$ & $\begin{array}{r}200 \\
120 \\
- \\
-\end{array}$ & $\begin{array}{l}- \\
- \\
- \\
-\end{array}$ & $\begin{array}{r}117 \\
- \\
- \\
-\end{array}$ & $\begin{array}{r}215 \\
- \\
- \\
-\end{array}$ & $\begin{array}{r}150 \\
100 \\
- \\
-\end{array}$ & $\begin{array}{r}140 \\
60 \\
- \\
-\end{array}$ & $\begin{array}{r}196 \\
118 \\
- \\
-\end{array}$ & $\begin{array}{r}235 \\
- \\
- \\
-\end{array}$ & 20 \\
\hline $\begin{array}{l}\text { Lead } \\
{\left[\mu \mathrm{g} / \mathrm{m}^{3}\right]}\end{array}$ & $\begin{array}{l}\text { Month } \\
\text { Quarter } \\
\text { Year }\end{array}$ & $\begin{array}{c}- \\
1.5\end{array}$ & 1.5 & 0.5 & $\begin{array}{r}- \\
- \\
0.5\end{array}$ & $\begin{array}{r}- \\
0.5\end{array}$ & $\begin{array}{r}- \\
- \\
0.5\end{array}$ & $\begin{array}{r}- \\
- \\
0.5\end{array}$ & $\begin{array}{l}- \\
-\end{array}$ & $\begin{array}{l}- \\
- \\
-\end{array}$ & $\begin{array}{r}- \\
- \\
0.5\end{array}$ & $\begin{array}{r}- \\
0.5\end{array}$ & 0.5 & $\begin{array}{l}- \\
- \\
-\end{array}$ & $\begin{array}{l}- \\
-\end{array}$ & $\begin{array}{l}- \\
- \\
2\end{array}$ & $\begin{array}{r}0.7 \\
- \\
-\end{array}$ & & $\begin{array}{l}- \\
- \\
-\end{array}$ & $\begin{array}{r}- \\
- \\
0.5\end{array}$ & $\begin{array}{l}- \\
-\end{array}$ & $\begin{array}{r}- \\
1.5 \\
1\end{array}$ & $\begin{array}{l}- \\
-\end{array}$ & $\begin{array}{r}- \\
- \\
0.75\end{array}$ & $\begin{array}{l}- \\
-\end{array}$ & $\begin{array}{r}- \\
1.5 \\
-\end{array}$ & $\begin{array}{r}- \\
0.2 \\
-\end{array}$ & $\begin{array}{r}- \\
1.5 \\
1\end{array}$ & $\begin{array}{r}- \\
- \\
0.5\end{array}$ & $\begin{array}{r}2.5 \\
- \\
-\end{array}$ & 1. \\
\hline $\begin{array}{l}\mathrm{PM}_{10} \\
{\left[\mu \mathrm{g} / \mathrm{m}^{3}\right]}\end{array}$ & $\begin{array}{l}1 \mathrm{hr} \\
24 \mathrm{hr} \\
\text { Annual }\end{array}$ & $\begin{array}{r}150 \\
50\end{array}$ & $\begin{array}{l}50 \\
20\end{array}$ & & $\begin{array}{r}50 \\
20^{*}\end{array}$ & $\begin{array}{l}50 \\
40\end{array}$ & $\begin{array}{l}50 \\
40\end{array}$ & $\begin{array}{l}50 \\
40\end{array}$ & - & 20 & $\begin{array}{r}50 \\
20^{*}\end{array}$ & $\begin{array}{r}- \\
50 \\
40\end{array}$ & $\begin{array}{l}50 \\
40\end{array}$ & $\begin{array}{l}- \\
- \\
-\end{array}$ & $\begin{array}{l}- \\
-\end{array}$ & $\begin{array}{r}- \\
50 \\
-\end{array}$ & - & & $\begin{array}{l}- \\
- \\
-\end{array}$ & $\begin{array}{r}- \\
50 \\
-\end{array}$ & $\begin{array}{r}- \\
150 \\
50\end{array}$ & $\begin{array}{r}- \\
150 \\
100\end{array}$ & $\begin{array}{r}- \\
70 \\
-\end{array}$ & $\begin{array}{r}- \\
100 \\
60\end{array}$ & $\begin{array}{r}200 \\
100 \\
-\end{array}$ & $\begin{array}{r}- \\
150 \\
50\end{array}$ & $\begin{array}{r}- \\
50 \\
20\end{array}$ & $\begin{array}{r}- \\
150 \\
60\end{array}$ & $\begin{array}{r}- \\
150 \\
70\end{array}$ & $\begin{array}{r}- \\
180 \\
60\end{array}$ & $\begin{array}{r}120 \\
50\end{array}$ \\
\hline $\begin{array}{l}\mathrm{PM}_{2.5} \\
{\left[\mu \mathrm{g} / \mathrm{m}^{3}\right]}\end{array}$ & $\begin{array}{l}24 \mathrm{hr} \\
\text { Annual }\end{array}$ & $\begin{array}{l}65 \\
15\end{array}$ & 12 & & - & - & - & - & - & - & - & - & & 30 & - & - & - & & - & - & - & - & - & - & - & - & - & - & - & - & \\
\hline $\begin{array}{l}\text { TSP } \\
{\left[\mu \mathrm{g} / \mathrm{m}^{3}\right]}\end{array}$ & $\begin{array}{l}24 \mathrm{hr} \\
\text { Annual }\end{array}$ & - & & & - & $\begin{array}{r}150 \\
-\end{array}$ & - & - & $\begin{array}{r}100 \\
50\end{array}$ & - & - & - & & $\begin{array}{r}120 \\
70\end{array}$ & $\begin{array}{r}100 \\
60\end{array}$ & $\begin{array}{r}200 \\
70\end{array}$ & $\begin{array}{r}120 \\
60\end{array}$ & $\begin{array}{r}150 \\
70\end{array}$ & $\begin{array}{r}- \\
150^{*}\end{array}$ & - & $\begin{array}{r}240 \\
80\end{array}$ & $\begin{array}{l}300 \\
200\end{array}$ & $\begin{array}{r}230 \\
90\end{array}$ & $\begin{array}{l}200 \\
140\end{array}$ & - & $\begin{array}{r}260 \\
75\end{array}$ & - & $\begin{array}{r}230 \\
90\end{array}$ & - & $\begin{array}{l}300 \\
100\end{array}$ & $\begin{array}{l}330 \\
100\end{array}$ \\
\hline $\begin{array}{l}\mathrm{SO}_{2} \\
{\left[\mu \mathrm{g} / \mathrm{m}^{3}\right]}\end{array}$ & $\begin{array}{l}1 \mathrm{hr} \\
24 \mathrm{hr} \\
\text { Annual }\end{array}$ & $\begin{array}{r}1300 \\
365 \\
80\end{array}$ & $\begin{array}{l}655 \\
105\end{array}$ & $\begin{array}{r}- \\
125 \\
50\end{array}$ & $\begin{array}{l}350 \\
125\end{array}$ & $\begin{array}{l}200 \\
120\end{array}$ & $\begin{array}{l}350 \\
125\end{array}$ & $\begin{array}{r}350 \\
125 \\
.\end{array}$ & $\begin{array}{r}- \\
150 \\
70\end{array}$ & $\begin{array}{r}- \\
125\end{array}$ & $\begin{array}{l}350 \\
125\end{array}$ & $\begin{array}{l}200 \\
100\end{array}$ & $\begin{array}{l}350 \\
125\end{array}$ & $\begin{array}{r}900 \\
300 \\
60\end{array}$ & $\begin{array}{r}450 \\
150 \\
30\end{array}$ & $\begin{array}{r}900 \\
260 \\
50\end{array}$ & $\begin{array}{r}690 \\
275 \\
55\end{array}$ & $\begin{array}{r}1310 \\
288 \\
52\end{array}$ & $\begin{array}{r}- \\
- \\
80^{*}\end{array}$ & $\begin{array}{r}520 \\
208 \\
52\end{array}$ & $\begin{array}{r}- \\
365 \\
80\end{array}$ & $\begin{array}{r}500 \\
150 \\
60\end{array}$ & $\begin{array}{r}350 \\
150 \\
60\end{array}$ & $\begin{array}{r}- \\
80 \\
60\end{array}$ & $\begin{array}{l}260 \\
104\end{array}$ & $\begin{array}{r}- \\
339 \\
80\end{array}$ & $\begin{array}{r}350 \\
120 \\
-\end{array}$ & $\begin{array}{r}- \\
180 \\
80\end{array}$ & $\begin{array}{r}390 \\
130 \\
52\end{array}$ & $\begin{array}{r}780 \\
260 \\
80\end{array}$ & $\begin{array}{l}780 \\
300 \\
100\end{array}$ \\
\hline
\end{tabular}

Notes: Red values are more stringent than the comparable U.S. NAAQS. Green values are less stringent. Standards for periods less than one hour are not shown.

Substances that are not subject to U.S. NAAQS (except Total Suspended Particulates) are not shown.

U.S. National Ambient Air Quality Standards ( ${ }^{*} 3$-hr secondary standard)

Calif. California is subject to U.S. NAAQS but promulgates some stricter standards. 
France Decree \#98-360, modified by decree \#2002-213. Limit values for human health, objective for ozone.

Germany Ordinance on Air Quality Standards, 1994. Working on new ordinance to implement EU directives.

Hungary Hungarian Standard MSz 21854/1990 \& 21854-1M/1994. Values listed are for "Protected I" zones.

Neth. Environmental quality objectives for 2010

Spain Real Decretos 1073/2002 \& 1796/2003. Limit values for human health, objective for ozone. ( ${ }^{*}$ final 2010 limit)

Sweden Ordinances 1997:693 \& 2001:527. Limit values for human health.

U.K. $\quad$ Air Quality Limit Values, separate but identical for each part of the U.K. (S.I. 2003/2121 for England). Target value for ozone.

Canada Standards listed are "max acceptable" values. "Max desirable" values are stricter, and "max tolerable" values are higher.

Alb. Ambient air quality guidelines.

B.C. Air quality objectives, 1995. Those listed are level B, "acceptable interim objectives."

Ont. Ambient air quality criteria.

Queb. Ambient air quality criteria.

Argentina National Law 20.284, 1973. Air quality standards. (* monthly standard)

Australia National Environment Protection Measure for Ambient Air Quality, 2003. Ambient air quality standards. ( ${ }^{*} 4$-hr standard)

Brazil National air quality standards, 1990

Egypt

PRC Ambient Air Quality Standard (GB-3095-1996) for residential areas.

Executive Regulations of Environmental Law no. 4, 1994

National Ambient Air Quality Standards, 1994. Those listed are for residential, rural, and other areas.

Environmental Quality Standards, $1973 \&$

Amial standards, 1993.

uidelines, 2002

Ambient Air Quality Guideline Values, 2000

Rep. Korea Air Quality Standards.

S. Africa SA has no promulgated AAQS, but one govt dept has published these guidelines.

Thailand Ambient air quality standards, 1995 
FIGURE S1: Summary of Air Standard Comparisons

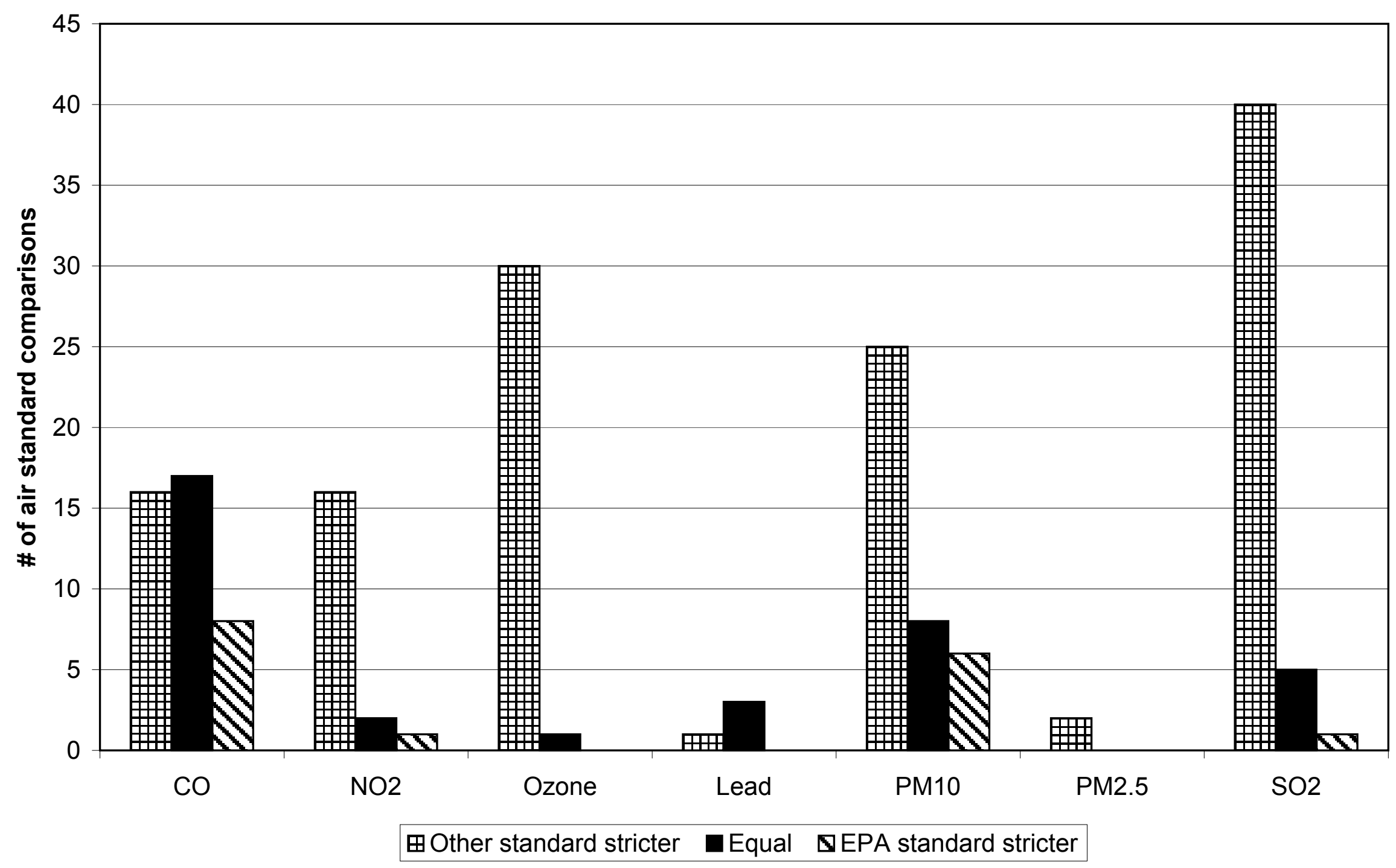


TABLE S2. Water Quality Standard Comparison - (a) Disinfectants and Disinfection Byproducts

\begin{tabular}{|c|c|c|c|c|c|c|c|c|c|c|c|c|c|c|c|c|c|c|}
\hline Contaminant $[\mathrm{mg} / \mathrm{l}]$ & $\stackrel{\text { ஸे }}{3}$ & 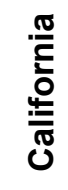 & 운 & 己 & 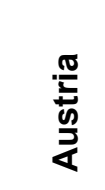 & 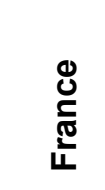 & 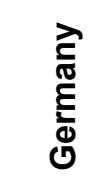 & |x & $\begin{array}{l}\frac{\pi}{0} \\
\frac{\pi}{\pi} \\
\frac{\pi}{\pi}\end{array}$ & $\begin{array}{l}\frac{\mathbb{m}}{\mathrm{E}} \\
\frac{\mathrm{g}}{\mathrm{Z}}\end{array}$ & $\underset{\dot{\infty}}{\dot{\infty}}$ & $\begin{array}{l}\stackrel{0}{\frac{0}{\pi}} \\
\stackrel{\text { In }}{0}\end{array}$ & $\begin{array}{l}0 \\
\mathbb{d} \\
\stackrel{0}{0} \\
\sigma \\
\sigma\end{array}$ & 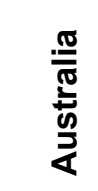 & $\begin{array}{l}\frac{1}{\pi} \\
\stackrel{0}{\pi} \\
\stackrel{\pi}{J}\end{array}$ & $\begin{array}{l}\stackrel{0}{0} \\
\frac{0}{X} \\
\Sigma\end{array}$ & $\underset{z}{\mathbf{N}}$ & 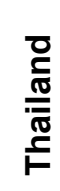 \\
\hline Bromate & 0.01 & & 0.025 & 0.01 & 0.01 & 0.01 & 0.01 & 0.01 & 0.01 & 0.01 & 0.01 & 0.01 & 0.01 & 0.02 & - & - & 0.025 & \\
\hline Chloramines (as $\mathrm{Cl}_{2}$ ) & 4 & & & - & & - & - & & 3 & 3 & 3 & 3 & 3 & - & - & - & - & - \\
\hline Chlorine & 4 & & 5 & - & & - & - & 1 & - & - & - & - & - & 5 & - & - & 5 & - \\
\hline Chlorine dioxide & 0.8 & & -1 & - & & - & - & -1 & - & - & - & - & 1 & 1 & - & - & - & 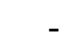 \\
\hline Chlorite & 1 & & 0.2 & - & & - & - & & - & - & - & - & & 0.3 & - & - & 0.3 & 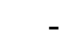 \\
\hline Haloacetic acids (HAA5) & 0.06 & & split & - & & - & - & - & - & - & - & - & $\ldots$ & split & - & - & split & - \\
\hline Trihalomethanes (TTHM) & 0.08 & & split & 0.1 & 0.03 & 0.1 & 0.05 & 0.1 & 0.1 & 0.1 & 0.1 & 0.1 & 0.08 & 0.25 & 0.1 & 0.2 & split & 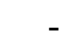 \\
\hline
\end{tabular}

Notes:

Red values are more stringent than the comparable U.S. NPDWR. Green values are less stringent.

split - standard is split among related chemicals

HAA5 is the sum of the concentrations of mono-, di-, and trichloroacetic acids and mono- and dibromoacetic acids.

TTHM is the sum of the concentrations of chloroform, bromodichloromethane, dibromochloromethane, and bromoform.

Substances that are not subject to U.S. NPDWR are not shown. 
TABLE S2. Water Quality Standard Comparison - (b) Organic Chemicals

\begin{tabular}{|c|c|c|c|c|c|c|c|c|c|c|c|c|c|c|c|c|c|c|}
\hline Contaminant $[\mu \mathrm{g} / \mathrm{l}]$ & $\stackrel{\oplus}{\supset}$ & 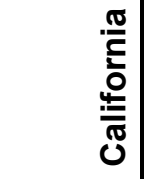 & 운 & 己 & 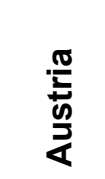 & $\begin{array}{l}\stackrel{\Xi}{0} \\
\frac{\pi}{\pi} \\
\text { L }\end{array}$ & 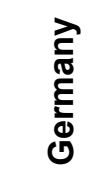 & |نّ & $\begin{array}{l}\frac{\pi}{0} \\
\frac{\pi}{\pi} \\
\frac{\pi}{\pi} \\
0\end{array}$ & $\begin{array}{l}\frac{\pi}{8} \\
\frac{0}{\mathbb{L}} \\
\end{array}$ & ن் & $\begin{array}{l}\stackrel{0}{\frac{0}{\pi}} \\
\stackrel{\text { In }}{\text { L }}\end{array}$ & $\begin{array}{l}0 \\
\mathbb{d} \\
\stackrel{0}{0} \\
\tilde{\sigma}\end{array}$ & $\begin{array}{l}\frac{\pi}{\sigma} \\
\frac{\pi}{\pi} \\
\frac{0}{2} \\
\frac{\pi}{4}\end{array}$ & $\begin{array}{l}\frac{c}{\pi} \\
\frac{0}{\pi} \\
\frac{\pi}{J}\end{array}$ & $\begin{array}{l}\stackrel{0}{x} \\
\frac{0}{x} \\
\Sigma\end{array}$ & $\stackrel{N}{\mathbf{z}}$ & 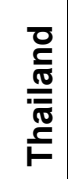 \\
\hline Acrylamide & TT & - & 0.5 & 0.1 & 0.1 & 0.1 & 0.1 & 0.1 & - & - & - & - & - & 0.2 & - & - & 0.5 & - \\
\hline Alachlor & 2 & 2 & 20 & $0.1^{*}$ & $0.1^{*}$ & $0.1^{*}$ & $0.1^{*}$ & $0.1^{*}$ & - & - & - & 5 & - & - & - & - & 20 & - \\
\hline Atrazine & 3 & 1 & 2 & $0.1^{*}$ & $0.1^{*}$ & $0.1^{*}$ & $0.1^{*}$ & $0.1^{*}$ & 5 & 5 & 5 & 5 & 5 & 20 & - & - & 2 & - \\
\hline Benzene & 5 & 1 & 10 & 1 & 1 & 1 & 1 & 1 & 5 & 5 & 5 & 5 & 5 & 1 & 10 & 10 & 10 & 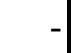 \\
\hline Benzo(a)pyrene & 0.2 & 0.2 & 0.7 & 0.01 & 0.01 & 0.01 & 0.01 & 0.01 & 0.01 & 0.01 & 0.01 & 0.01 & 0.01 & 0.01 & - & - & 0.7 & - \\
\hline Carbofuran & 40 & 18 & 7 & $0.1^{*}$ & $0.1^{*}$ & $0.1^{*}$ & $0.1^{*}$ & $0.1^{*}$ & 90 & 90 & 90 & 90 & 90 & 10 & - & - & 8 & - \\
\hline Carbon Tetrachloride & 5 & 0.5 & 2 & - & - & - & - & - & 5 & 5 & 5 & 5 & 5 & 3 & 2 & - & 2 & - \\
\hline Chlordane & 2 & 0.1 & 0.2 & $0.1^{*}$ & $0.1^{*}$ & $0.1^{*}$ & $0.1^{*}$ & $0.1^{*}$ & - & - & - & 7 & - & 1 & - & 0.2 & 0.2 & - \\
\hline Chlorobenzene & 100 & 70 & 300 & - & - & - & - & - & 80 & 80 & 80 & 80 & 80 & 300 & - & - & 300 & - \\
\hline 2,4-D & 70 & 70 & 30 & $0.1^{*}$ & $0.1^{*}$ & $0.1^{*}$ & $0.1^{*}$ & $0.1^{*}$ & 100 & 100 & 100 & 100 & 100 & 30 & - & 30 & 40 & - \\
\hline Dalapon & 200 & 200 & - & $0.1^{*}$ & $0.1^{*}$ & $0.1^{*}$ & $0.1^{*}$ & $0.1^{*}$ & - & - & - & - & -1 & 500 & - & - & - & - \\
\hline DBCP & 0.2 & 0.2 & 1 & $0.1^{*}$ & $0.1^{*}$ & $0.1^{*}$ & $0.1^{*}$ & $0.1^{*}$ & - & - & - & - & - & - & - & - & 1 & - \\
\hline 1,2(o)-Dichlorobenzene & 600 & 600 & 1000 & - & - & - & - & - & 200 & 200 & 200 & 200 & 200 & 1500 & - & - & 1000 & - \\
\hline 1,4(p)-Dichlorobenzene & 75 & 5 & 300 & - & - & - & - & - & 5 & 5 & 5 & 5 & 5 & 40 & - & - & 400 & - \\
\hline 1,2-Dichloroethane & 5 & 0.5 & 30 & 3 & 3 & 3 & 3 & 3 & 5 & 5 & 5 & 5 & 5 & 3 & 4 & - & 30 & - \\
\hline 1,1-Dichloroethylene & 7 & 6 & 30 & - & - & - & - & - & 14 & 14 & 14 & 14 & 14 & 30 & 20 & - & 30 & - \\
\hline cis-1,2-Dichloroethylene & 70 & 6 & 50 & - & - & - & - & - & - & - & - & - & - & 60 & 40 & - & 60 & - \\
\hline trans-1,2-Dichloroethylene & 100 & 10 & incl & - & - & - & - & - & - & - & - & - & - & incl & - & - & incl & - \\
\hline Dichloromethane & 5 & 5 & 20 & - & - & - & - & & 50 & 50 & 50 & 50 & 50 & 4 & 20 & - & 20 & - \\
\hline 1,2-Dichloropropane & 5 & 5 & 40 & - & - & - & - & - & - & - & - & - & - & - & - & - & 20 & - \\
\hline Di(2-ethylhexyl) adipate & 400 & 400 & 80 & - & - & - & - & - & - & - & - & - & - & - & - & - & 100 & - \\
\hline Di(2-ethylhexyl) phthalate & 6 & 4 & 8 & - & - & - & - & - & - & - & - & - & - & 10 & - & - & 9 & - \\
\hline Dinoseb & 7 & 7 & - & $0.1^{*}$ & $0.1^{*}$ & $0.1^{*}$ & $0.1^{*}$ & $0.1^{*}$ & 10 & 10 & 10 & 10 & 10 & - & - & - & - & - \\
\hline Dioxin & 0.00003 & 0.00003 & -1 & - & - & - & - & - & - & - & - & $1.5 \mathrm{E}-05$ & -1 & - & - & - & - & - \\
\hline Diquat & 20 & 20 & 10 & $0.1^{*}$ & $0.1^{*}$ & $0.1^{*}$ & $0.1^{*}$ & $0.1^{*}$ & 70 & 70 & 70 & 70 & 70 & 5 & - & - & 10 & - \\
\hline Endothall & 100 & 100 & & $0.1^{*}$ & $0.1^{*}$ & $0.1^{*}$ & $0.1^{*}$ & $0.1^{*}$ & - & - & - & - & - & 100 & - & - & - & - \\
\hline Endrin & 2 & 2 & - & $0.1^{*}$ & $0.1^{*}$ & $0.1^{*}$ & $0.1^{*}$ & $0.1^{*}$ & - & - & - & - & - & - & - & - & - & - \\
\hline Epichlorohydrin & TT & & 0.4 & 0.1 & 0.1 & 0.1 & 0.1 & 0.1 & - & - & - & - & - & 0.5 & - & - & 0.5 & 1 \\
\hline Ethylbenzene & 700 & 300 & 300 & - & - & - & - & - & - & - & - & - & - & 300 & - & 300 & 300 & - \\
\hline Ethylene dibromide & 0.05 & 0.05 & 1 & $0.1^{*}$ & $0.1^{*}$ & $0.1^{*}$ & $0.1^{*}$ & $0.1^{*}$ & - & - & - & - & - & 1 & - & - & - & - \\
\hline Glyphosate & 700 & 700 & & $0.1^{*}$ & $0.1^{*}$ & $0.1^{*}$ & $0.1^{*}$ & $0.1^{*}$ & 280 & 280 & 280 & 280 & 280 & 1000 & - & - & - & - \\
\hline Heptachlor & 0.4 & 0.01 & 0.03 & 0.03 & 0.03 & 0.03 & 0.03 & 0.03 & - & - & - & 3 & -1 & 0.3 & - & 0.03 & 0.04 & 1 \\
\hline
\end{tabular}




\begin{tabular}{|c|c|c|c|c|c|c|c|c|c|c|c|c|c|c|c|c|c|}
\hline Heptachlor epoxide & 0.2 & 0.01 & incl & 0.03 & 0.03 & 0.03 & 0.03 & 0.03 & - & - & - & incl & - & incl & - & incl & incl \\
\hline Hexachlorobenzene & 1 & 1 & 1 & $0.1^{*}$ & $0.1^{*}$ & $0.1^{*}$ & $0.1^{*}$ & $0.1 *$ & - & - & - & - & - & - & - & 1 & 1 \\
\hline Hexachlorocyclopentadiene & 50 & 50 & - & - & - & - & - & & - & - & - & - & - & - & - & - & - \\
\hline Lindane & 0.2 & 0.2 & 2 & $0.1^{*}$ & $0.1^{*}$ & $0.1^{*}$ & $0.1^{*}$ & $0.1^{*}$ & - & - & - & 4 & ـ & 20 & - & 2 & 2 \\
\hline Methoxychlor & 40 & 30 & 20 & $0.1^{*}$ & $0.1^{*}$ & $0.1^{*}$ & $0.1^{*}$ & $0.1^{*}$ & 900 & 900 & 900 & 900 & 900 & 300 & - & 20 & 20 \\
\hline Oxamyl (Vydate) & 200 & 50 & - & $0.1^{*}$ & $0.1^{*}$ & $0.1^{*}$ & $0.1^{*}$ & $0.1 *$ & - & - & - & - & -1 & 100 & - & - & - \\
\hline Pentachlorophenol & 1 & 1 & 9 & - & - & - & - & & 60 & 60 & 60 & 60 & 60 & 10 & - & - & 10 \\
\hline Picloram & 500 & 500 & - & $0.1^{*}$ & $0.1^{*}$ & $0.1^{*}$ & $0.1^{*}$ & $0.1^{*}$ & 190 & 190 & 190 & 190 & 190 & 300 & - & - & 200 \\
\hline PCBs & 0.5 & 0.5 & - & & - & - & - & & - & - & - & 3 & & - & - & - & - \\
\hline Simazine & 4 & 4 & 2 & $0.1^{*}$ & $0.1^{*}$ & $0.1^{*}$ & $0.1^{*}$ & $0.1^{*}$ & 10 & 10 & 10 & 10 & 10 & 20 & 3 & - & 2 \\
\hline Styrene & 100 & 100 & 20 & - & - & - & - & & - & - & - & - & & 30 & - & - & 30 \\
\hline Tetrachloroethylene & 5 & 5 & 40 & 10 & 10 & 10 & 10 & 10 & 30 & 30 & 30 & 30 & 30 & 50 & 10 & - & 50 \\
\hline Toluene & 1000 & 150 & 700 & - & - & - & - & - & - & - & - & - & -1 & 800 & - & 700 & 800 \\
\hline Toxaphene & 3 & 3 & - & $0.1^{*}$ & $0.1^{*}$ & $0.1^{*}$ & $0.1^{*}$ & $0.1 *$ & - & - & - & - & -1 & - & - & - & - \\
\hline 2,4,5-TP (Silvex) & 50 & 50 & 9 & $0.1^{*}$ & $0.1^{*}$ & $0.1^{*}$ & $0.1^{*}$ & $0.1^{*}$ & - & - & - & 280 & - & 10 & - & - & 10 \\
\hline 1,2,4-Trichlorobenzene & 70 & 5 & 20 & - & - & - & - & & - & - & - & - & -1 & 30 & - & - & 30 \\
\hline 1,1,1-Trichloroethane & 200 & 200 & 2000 & - & - & - & - & - & - & - & - & - & -1 & - & - & - & 200 \\
\hline 1,1,2-Trichloroethane & 5 & 5 & & - & - & - & - & & - & - & - & - & - & - & 6 & - & - \\
\hline Trichloroethylene & 5 & 5 & 70 & incl & incl & incl & incl & incl & 50 & 50 & 50 & 50 & 50 & - & 30 & - & 80 \\
\hline Vinyl chloride & 2 & 0.5 & 5 & 0.5 & 0.5 & 0.5 & 0.5 & 0.5 & 2 & 2 & 2 & 2 & 2 & 0.3 & - & - & 5 \\
\hline Xylenes (total) & 10000 & 1750 & 500 & - & - & - & - & - & - & - & - & - & - & 600 & - & 500 & 600 \\
\hline
\end{tabular}

Notes:

Red values are more stringent than the comparable U.S. NPDWR. Green values are less stringent. incl - included in standard for related chemical

Substances that are not subject to U.S. NPDWR are not shown.

U.S. National Primary Drinking Water Regulations. TT - treatment technique required.

Calif. California is subject to U.S. NPDWR but also promulgates its own standards, some stricter.

WHO Guidelines for Drinking Water Quality, 1996 \& 1998.

EU $\quad$ EU Council Directive 98/83/EC. Member States must comply in 5 years.

* Individual pesticide limit $=0.1 \mu \mathrm{g} / \mathrm{l}$. Total pesticide limit $=0.5 \mu \mathrm{g} / \mathrm{l}$.

Austria Drinking Water Regulation, 2001

France Decree \#2001-1220.

Germ. Drinking Water Regulation, 2001.

U.K. Water Quality Regulations, separate but identical for each part of the U.K. (S.I. 2000/3184 \& 2001/2885 for England)

Canada Guidelines for Canadian Drinking Water Quality 2001

Outside federal land, provinces can enact their own drinking water laws and regulations. 
Alb. Adopted the Canadian guidelines as legally enforceable standards.

B.C. Safe Drinking Water Regulations - apparently not enforced.

Ont. Drinking-Water Quality Standards. Ontario Regulation 169/03, amended to 17/04.

Que. Regulation respecting the quality of drinking water, updated to 2004

Australia Guidelines - authoritative reference. Not mandatory. Also list many other pesticides.

Pesticide values listed are "health values," not the lower "guideline values.

Japan Quality standards, 1993.

Mexico Official standards set 1994, revised 2000

N.Z. Drinking water standards, 2000

Thailand Drinking water quality standards, 1978 
FIGURE S2: Summary of Water Standard Comparisons

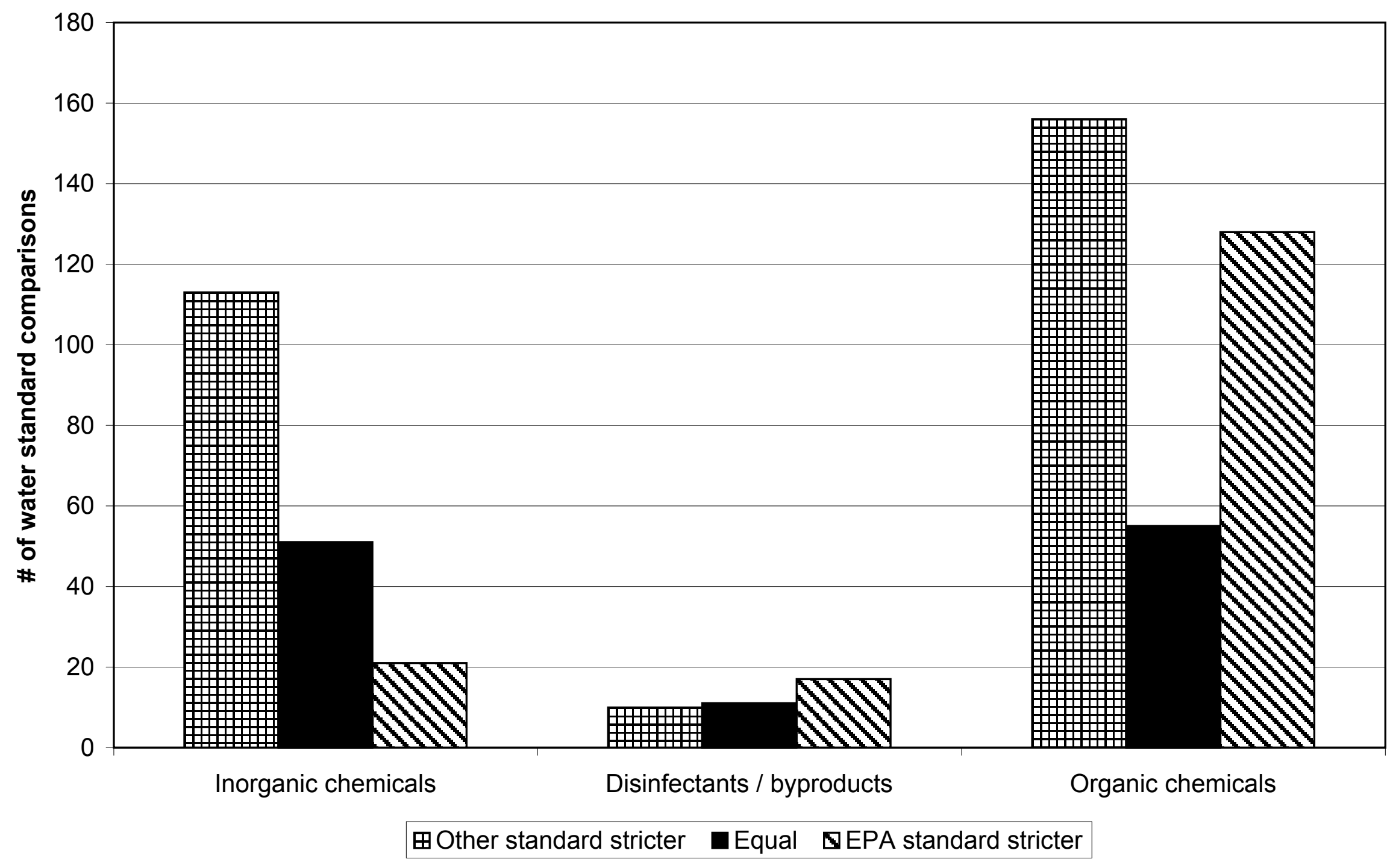




\begin{tabular}{|c|c|c|c|c|c|}
\hline \multirow[b]{2}{*}{ Substance } & \multicolumn{5}{|c|}{ Reference values $\left[\mathrm{mg} / \mathrm{m}^{3}\right]^{a}$} \\
\hline & $\mathrm{EPA}^{\mathrm{b}}$ & ATSDR $^{\mathrm{C}}$ & California $^{d}$ & Canada $^{\mathrm{e}}$ & Neth. $^{f}$ \\
\hline Acetaldehyde & $9 \times 10^{-3}$ & - & $9 \times 10^{-3}$ & $4 \times 10^{-1}$ & - \\
\hline Acrolein & $2 \times 10^{-5}$ & - & $6 \times 10^{-5}$ & $4 \times 10^{-4}$ & - \\
\hline Acrylonitrile & $2 \times 10^{-3}$ & - & $5 \times 10^{-3}$ & - & - \\
\hline Ammonia & $1 \times 10^{-1}$ & $2 \times 10^{-1}$ & $2 \times 10^{-1}$ & - & - \\
\hline Benzene & $3 \times 10^{-2}$ & - & $6 \times 10^{-2}$ & - & - \\
\hline Bromomethane & $5 \times 10^{-3}$ & $2 \times 10^{-2}$ & $5 \times 10^{-3}$ & - & - \\
\hline Carbon disulfide & $7 \times 10^{-1}$ & $8 \times 10^{-1}$ & - & $1 \times 10^{-1}$ & - \\
\hline Chlordane & $7 \times 10^{-4}$ & $2 \times 10^{-5}$ & - & - & - \\
\hline Chlorine dioxide & $2 \times 10^{-4}$ & - & $6 \times 10^{-4}$ & - & - \\
\hline 1,4-Dichlorobenzene & $8 \times 10^{-1}$ & $6 \times 10^{-1}$ & $8 \times 10^{-1}$ & - & $7 \times 10^{-1}$ \\
\hline 1,3-Dichloropropene & $2 \times 10^{-2}$ & $9 \times 10^{-3}$ & - & - & - \\
\hline Dichlorvos & $5 \times 10^{-4}$ & $5 \times 10^{-4}$ & - & - & - \\
\hline Diesel engine emissions & $5 \times 10^{-3}$ & - & $5 \times 10^{-3}$ & - & - \\
\hline $\mathrm{N}, \mathrm{N}$-Dimethylformamide & $3 \times 10^{-2}$ & - & $8 \times 10^{-2}$ & $1 \times 10^{-1}$ & - \\
\hline Epichlorohydrin & $1 \times 10^{-3}$ & - & $3 \times 10^{-3}$ & - & - \\
\hline 1,2-Epoxybutane (EBU) & $2 \times 10^{-2}$ & - & $2 \times 10^{-2}$ & - & - \\
\hline Ethyl chloride & $1 \times 10^{1}$ & - & $3 \times 10^{1}$ & - & - \\
\hline Ethylbenzene & $1 \times 10^{0}$ & - & $2 \times 10^{0}$ & - & $8 \times 10^{-1}$ \\
\hline Ethylene glycol monobutyl ether (EGBE) & $1 \times 10^{1}$ & $1 \times 10^{0}$ & - & $1 \times 10^{1}$ & - \\
\hline Hexachlorocyclopentadiene (HCCPD) & $2 \times 10^{-4}$ & $3 \times 10^{-3}$ & - & - & - \\
\hline 1,6-Hexamethylene diisocyanate & $1 \times 10^{-5}$ & $7 \times 10^{-5}$ & - & - & - \\
\hline n-Hexane & $2 \times 10^{-1}$ & $2 \times 10^{0}$ & $7 \times 10^{0}$ & - & - \\
\hline Hydrogen chloride & $2 \times 10^{-2}$ & - & $9 \times 10^{-3}$ & - & - \\
\hline Hydrogen cyanide & $3 \times 10^{-3}$ & - & $9 \times 10^{-3}$ & - & - \\
\hline Hydrogen sulfide & $2 \times 10^{-3}$ & - & $1 \times 10^{-2}$ & - & - \\
\hline Manganese & $5 \times 10^{-5}$ & $4 \times 10^{-5}$ & $2 \times 10^{-4}$ & - & - \\
\hline Mercury, elemental & $3 \times 10^{-4}$ & $2 \times 10^{-4}$ & $9 \times 10^{-5}$ & - & $2 \times 10^{-4}$ \\
\hline Methyl chloride & $9 \times 10^{-2}$ & $1 \times 10^{-1}$ & - & - & - \\
\hline Methyl methacrylate & $7 \times 10^{-1}$ & - & - & $5 \times 10^{-2}$ & - \\
\hline Methyl tert-butyl ether (MTBE) & $3 \times 10^{0}$ & $3 \times 10^{0}$ & - & $4 \times 10^{-2}$ & - \\
\hline Methylene diphenyl diisocyanate & $6 \times 10^{-4}$ & - & $7 \times 10^{-4}$ & - & - \\
\hline Naphthalene & $3 \times 10^{-3}$ & $7 \times 10^{-4}$ & $9 \times 10^{-3}$ & - & - \\
\hline Phosphoric acid & $1 \times 10^{-2}$ & - & $7 \times 10^{-3}$ & - & - \\
\hline Propylene glycol monomethyl ether & $2 \times 10^{0}$ & - & $7 \times 10^{0}$ & - & - \\
\hline Propylene oxide & $3 \times 10^{-2}$ & - & $3 \times 10^{-2}$ & - & - \\
\hline Styrene & $1 \times 10^{0}$ & $3 \times 10^{-1}$ & $9 \times 10^{-1}$ & $9 \times 10^{-2}$ & $9 \times 10^{-1}$ \\
\hline Toluene & $4 \times 10^{-1}$ & $3 \times 10^{-1}$ & $3 \times 10^{-1}$ & $4 \times 10^{0}$ & $4 \times 10^{-1}$ \\
\hline 2,4-/2,6-Toluene diisocyanate mixture & $7 \times 10^{-5}$ & - & $7 \times 10^{-5}$ & - & - \\
\hline Vinyl acetate & $2 \times 10^{-1}$ & - & $2 \times 10^{-1}$ & - & - \\
\hline Xylenes & $1 \times 10^{-1}$ & $4 \times 10^{-1}$ & $7 \times 10^{-1}$ & $2 \times 10^{-1}$ & $9 \times 10^{-1}$ \\
\hline
\end{tabular}

a A lower non-cancer reference value indicates a higher estimated toxicity or a greater degree of uncertainty. Red values indicate toxicity estimates higher than EPA's. Green values indicate lower.

b EPA values are Reference Concentrations from the Integrated Risk Information System. 
${ }^{c}$ ATSDR values are Minimal Risk Levels from published toxicity profiles.

d California values are Chronic Reference Exposure Levels from the Toxicity Criteria Database.

e Health Canada values are Tolerable Concentrations from the ITER database.

${ }^{f}$ Netherlands values are Tolerable Concentrations from RIVM report 711701025. 
TABLE S4. Oral Non-Cancer Health Assessment Comparison

Reference values [mg/kg-day] ${ }^{\mathrm{a}}$

\begin{tabular}{|c|c|c|c|c|c|}
\hline \multirow[b]{2}{*}{ Substance } & & & & & \\
\hline & $\mathrm{EPA}^{\mathrm{b}}$ & ATSDR $^{c}$ & California $^{d}$ & Canada $^{\mathrm{e}}$ & Neth. ${ }^{f}$ \\
\hline Acrolein & $5 \times 10^{-4}$ & $5 \times 10^{-4}$ & - & $7.5 \times 10^{-3}$ & - \\
\hline Aldrin & $3 \times 10^{-5}$ & $3 \times 10^{-5}$ & - & - & $1 \times 10^{-4}$ \\
\hline Anthracene & $3 \times 10^{-1}$ & - & - & - & $4 \times 10^{-2}$ \\
\hline Aroclor 1254 & $2 \times 10^{-5}$ & $2 \times 10^{-5}$ & - & - & - \\
\hline Arsenic, inorganic & $3 \times 10^{-4 \mathrm{~g}}$ & $3 \times 10^{-4}$ & - & - & $1 \times 10^{-3}$ \\
\hline Atrazine & $3.5 \times 10^{-2}$ & - & - & - & $5 \times 10^{-3}$ \\
\hline Barium and compounds & $7 \times 10^{-2}$ & - & - & - & $2 \times 10^{-2}$ \\
\hline Beryllium and compounds & $2 \times 10^{-3}$ & $2 \times 10^{-3}$ & - & - & - \\
\hline Bromodichloromethane & $2 \times 10^{-2}$ & $2 \times 10^{-2}$ & - & - & - \\
\hline Bromoform & $2 \times 10^{-2}$ & $2 \times 10^{-1}$ & - & - & - \\
\hline Butyl benzyl phthalate & $2 \times 10^{-1}$ & - & - & $1 \times 10^{0}$ & $5 \times 10^{-1}$ \\
\hline Cadmium & $5 \times 10^{-4}$ & $2 \times 10^{-4}$ & - & - & $5 \times 10^{-4}$ \\
\hline Carbaryl & $1 \times 10^{-1}$ & - & - & - & $3 \times 10^{-3}$ \\
\hline Carbofuran & $5 \times 10^{-3}$ & - & - & - & $2 \times 10^{-3}$ \\
\hline Chlordane & $5 \times 10^{-4}$ & $6 \times 10^{-4}$ & - & - & - \\
\hline Chlorobenzene & $2 \times 10^{-2}$ & - & - & $4 \times 10^{-1}$ & $2 \times 10^{-1}$ \\
\hline Chloroform & $1 \times 10^{-2}$ & $1 \times 10^{-2}$ & - & - & $3 \times 10^{-2}$ \\
\hline beta-Chloronaphthalene & $8 \times 10^{-2}$ & - & - & - & $8 \times 10^{-2}$ \\
\hline 2-Chlorophenol & $5 \times 10^{-3}$ & - & - & - & $3 \times 10^{-3}$ \\
\hline Chlorpyrifos & $3 \times 10^{-3}$ & $1 \times 10^{-3}$ & - & - & - \\
\hline Chromium(III), insoluble salts & $1.5 \times 10^{0}$ & - & - & - & $5 \times 10^{0}$ \\
\hline Cyanide, free & $2 \times 10^{-2}$ & - & - & - & $5 \times 10^{-2}$ \\
\hline Cyclohexanone & $5 \times 10^{0}$ & - & - & - & $5 \times 10^{0}$ \\
\hline Di(2-ethylhexyl)phthalate & $2 \times 10^{-2}$ & $6 \times 10^{-2}$ & - & - & $4 \times 10^{-3}$ \\
\hline Dibromochloromethane & $2 \times 10^{-2}$ & $9 \times 10^{-2}$ & - & - & - \\
\hline Dibutyl phthalate & $1 \times 10^{-1}$ & - & - & $6 \times 10^{-2}$ & $5 \times 10^{-2}$ \\
\hline 1,2-Dichlorobenzene & $9 \times 10^{-2}$ & - & - & $4 \times 10^{-1}$ & $4 \times 10^{-1}$ \\
\hline p,p'-Dichlorodiphenyl trichloroethane & $5 \times 10^{-4}$ & - & - & - & $5 \times 10^{-4}$ \\
\hline trans-1,2-Dichloroethylene & $2 \times 10^{-2}$ & - & - & - & $2 \times 10^{-2}$ \\
\hline 1,1-Dichloroethylene & $5 \times 10^{-2}$ & $9 \times 10^{-3}$ & - & - & - \\
\hline Dichloromethane & $6 \times 10^{-2}$ & $6 \times 10^{-2}$ & - & $5 \times 10^{-2}$ & $6 \times 10^{-2}$ \\
\hline 2,4-Dichlorophenol & $3 \times 10^{-3}$ & - & - & - & $3 \times 10^{-3}$ \\
\hline Dichlorvos & $5 \times 10^{-4}$ & $5 \times 10^{-4}$ & - & - & - \\
\hline Dieldrin & $5 \times 10^{-5}$ & $5 \times 10^{-5}$ & - & - & $1 \times 10^{-4}$ \\
\hline Diisopropyl methylphosphonate & $8 \times 10^{-2}$ & $6 \times 10^{-1}$ & - & - & - \\
\hline 2,4-Dinitrotoluene & $2 \times 10^{-3}$ & $2 \times 10^{-3}$ & - & - & - \\
\hline Disulfoton & $4 \times 10^{-5}$ & $6 \times 10^{-5}$ & - & - & - \\
\hline Endosulfan & $6 \times 10^{-3}$ & $2 \times 10^{-3}$ & - & - & - \\
\hline Endrin & $3 \times 10^{-4}$ & $3 \times 10^{-4}$ & - & - & $2 \times 10^{-4}$ \\
\hline Ethion & $5 \times 10^{-4}$ & $4 \times 10^{-4}$ & - & - & - \\
\hline Ethylbenzene & $1 \times 10^{-1}$ & - & - & - & $1 \times 10^{-1}$ \\
\hline Ethylene glycol & $2 \times 10^{0}$ & $2 \times 10^{0}$ & - & $5 \times 10^{-2}$ & - \\
\hline
\end{tabular}




\begin{tabular}{|c|c|c|c|c|c|}
\hline Fluorene & $4 \times 10^{-2}$ & - & - & - & $4 \times 10^{-2}$ \\
\hline Fluorine (soluble fluoride) & $6 \times 10^{-2}$ & $5 \times 10^{-2}$ & - & $2 \times 10^{-1}$ & - \\
\hline Formaldehyde & $2 \times 10^{-1}$ & $2 \times 10^{-1}$ & - & $2.6 \mathrm{mg} / \mathrm{l}$ & - \\
\hline Hexachlorobenzene & $8 \times 10^{-4}$ & $5 \times 10^{-5}$ & - & $5 \times 10^{-4}$ & - \\
\hline gamma-Hexachlorocyclohexane & $3 \times 10^{-4}$ & - & - & - & $4 \times 10^{-5}$ \\
\hline Isophorone & $2 \times 10^{-1}$ & $2 \times 10^{-1}$ & - & - & - \\
\hline Malathion & $2 \times 10^{-2}$ & $2 \times 10^{-2}$ & - & - & - \\
\hline Maneb & $5 \times 10^{-3}$ & - & - & - & $5 \times 10^{-2}$ \\
\hline Methyl methacrylate & $1 \times 10^{0}$ & - & - & $5 \times 10^{-2}$ & - \\
\hline Methyl parathion & $2.5 \times 10^{-4}$ & $3 \times 10^{-4}$ & - & - & - \\
\hline Methylmercury (MeHg) & $1 \times 10^{-4}$ & $3 \times 10^{-4}$ & - & - & $1 \times 10^{-4}$ \\
\hline 2-Methylnaphthalene & $4 \times 10^{-3}$ & $5 \times 10^{-2}$ & - & - & - \\
\hline Mirex & $2 \times 10^{-4}$ & $8 \times 10^{-4}$ & - & - & - \\
\hline Molybdenum & $5 \times 10^{-3}$ & - & - & - & $1 \times 10^{-2}$ \\
\hline Naphthalene & $2 \times 10^{-2}$ & - & - & - & $4 \times 10^{-2}$ \\
\hline Nickel, soluble salts & $2 \times 10^{-2}$ & - & - & $5 \times 10^{-2}$ & $5 \times 10^{-2}$ \\
\hline Pentachlorobenzene & $8 \times 10^{-4}$ & - & - & $1 \times 10^{-3}$ & - \\
\hline Pentachlorophenol & $3 \times 10^{-2}$ & $1 \times 10^{-3}$ & - & - & $3 \times 10^{-3}$ \\
\hline Phenol & $3 \times 10^{-1}$ & - & - & $1 \times 10^{-1}$ & $4 \times 10^{-2}$ \\
\hline Pyridine & $1 \times 10^{-3}$ & - & - & - & $1 \times 10^{-3}$ \\
\hline Selenium and Compounds & $5 \times 10^{-3}$ & $5 \times 10^{-3}$ & - & - & - \\
\hline Styrene & $2 \times 10^{-1}$ & - & - & $1 \times 10^{-1}$ & $1 \times 10^{-1}$ \\
\hline 1,2,4,5-Tetrachlorobenzene & $3 \times 10^{-4}$ & - & - & $2 \times 10^{-4}$ & - \\
\hline Tetrachloroethylene & $1 \times 10^{-2}$ & - & - & $1 \times 10^{-2}$ & $2 \times 10^{-2}$ \\
\hline 2,3,4,6-Tetrachlorophenol & $3 \times 10^{-2}$ & - & - & - & $3 \times 10^{-3}$ \\
\hline Toluene & $2 \times 10^{-1}$ & - & - & $2 \times 10^{-1}$ & $2 \times 10^{-1}$ \\
\hline 1,2,4-Trichlorobenzene & $1 \times 10^{-2}$ & - & - & $2 \times 10^{-3}$ & $8 \times 10^{-3}$ \\
\hline 2,4,5-Trichlorophenol & $1 \times 10^{-1}$ & - & - & - & $3 \times 10^{-3}$ \\
\hline Vinyl chloride & $3 \times 10^{-3}$ & $2 \times 10^{-5}$ & - & - & - \\
\hline Xylenes & $2 \times 10^{-1}$ & - & - & $1.5 \times 10^{0}$ & $1.5 \times 10^{-1}$ \\
\hline Zinc and Compounds & $3 \times 10^{-1}$ & $3 \times 10^{-1}$ & - & - & $5 \times 10^{-1}$ \\
\hline
\end{tabular}

a A lower non-cancer reference value indicates a higher estimated toxicity or a greater degree of uncertainty. Red values indicate toxicity estimates higher than EPA's. Green values indicate lower.

b EPA values are Reference Doses from the Integrated Risk Information System.

c ATSDR values are Minimal Risk Levels from published toxicity profiles.

d California does not list oral Chronic Reference Exposure Levels in their Toxicity Criteria Database.

e Health Canada values are Tolerable Daily Intakes from the ITER database.

${ }^{f}$ Netherlands values are Tolerable Daily Intakes from RIVM report 711701025.

${ }^{g}$ This IRIS estimate is not the basis for the current MCL for arsenic in drinking water. 
TABLE S5. Inhalation Cancer Potency Assessment Comparison

Risk-specific concentrations $\left[\mathrm{mg} / \mathrm{m}^{3}\right]^{a}$

\begin{tabular}{|c|c|c|c|c|}
\hline \multirow{2}{*}{ Substance } & \multirow{2}{*}{$E A^{b}$} & \multirow[b]{2}{*}{ California $^{c}$} & \multirow{2}{*}{ Canada $^{d}$} & \multirow{2}{*}{ Neth. ${ }^{\mathrm{e}}$} \\
\hline & & & & \\
\hline Acetaldehyde & $5 \times 10^{-3}$ & $4 \times 10^{-3}$ & - & - \\
\hline Acrylamide & $8 \times 10^{-6}$ & $8 \times 10^{-6}$ & - & - \\
\hline Acrylonitrile & $1 \times 10^{-4}$ & $3 \times 10^{-5}$ & $1 \times 10^{-3}$ & - \\
\hline Aldrin & $2 \times 10^{-6}$ & $2 \times 10^{-6}$ & - & - \\
\hline Aramite & $1 \times 10^{-3}$ & $1 \times 10^{-3}$ & - & - \\
\hline Arsenic, inorganic & $2 \times 10^{-6}$ & $3 \times 10^{-6}$ & $2 \times 10^{-6}$ & - \\
\hline Azobenzene & $3 \times 10^{-4}$ & $3 \times 10^{-4}$ & - & - \\
\hline Benzene & $1 \times 10^{-3 f}$ & $3 \times 10^{-4}$ & $3 \times 10^{-3}$ & $2 \times 10^{-3}$ \\
\hline Benzidine & $1 \times 10^{-7}$ & $7 \times 10^{-8}$ & - & - \\
\hline Beryllium and compounds & $4 \times 10^{-6}$ & $4 \times 10^{-6}$ & - & - \\
\hline Bis(chloroethyl)ether (BCEE) & $3 \times 10^{-5}$ & $1 \times 10^{-5}$ & - & - \\
\hline Bis(chloromethyl)ether (BCME) & $2 \times 10^{-7}$ & $8 \times 10^{-7}$ & $1 \times 10^{-6}$ & - \\
\hline 1,3-Butadiene & $3 \times 10^{-4}$ & $6 \times 10^{-5}$ & $2 \times 10^{-3}$ & - \\
\hline Cadmium & $6 \times 10^{-6}$ & $2 \times 10^{-6}$ & $1 \times 10^{-6}$ & - \\
\hline Carbon tetrachloride & $7 \times 10^{-4}$ & $2 \times 10^{-4}$ & - & - \\
\hline Chlordane & $1 \times 10^{-4}$ & $3 \times 10^{-5}$ & - & - \\
\hline Chloroform & $4 \times 10^{-4}$ & $2 \times 10^{-3}$ & - & - \\
\hline Chromium(VI) & $8 \times 10^{-7}$ & $7 \times 10^{-8}$ & $1 \times 10^{-7}$ & $2 \times 10^{-7}$ \\
\hline Coke oven emissions & $2 \times 10^{-5}$ & $2 \times 10^{-5}$ & - & - \\
\hline 1,2-Dibromoethane & $5 \times 10^{-5}$ & $1 \times 10^{-4}$ & - & - \\
\hline p,p'-Dichlorodiphenyltrichloroethane (DDT) & $1 \times 10^{-4}$ & $1 \times 10^{-4}$ & - & - \\
\hline 1,2-Dichloroethane & $4 \times 10^{-4}$ & $5 \times 10^{-4}$ & - & - \\
\hline Dichloromethane & $2 \times 10^{-2}$ & $1 \times 10^{-2}$ & $4 \times 10^{-1}$ & - \\
\hline 1,3-Dichloropropene & $2 \times 10^{-3}$ & $6 \times 10^{-4}$ & - & - \\
\hline Dieldrin & $2 \times 10^{-6}$ & $2 \times 10^{-6}$ & - & - \\
\hline 1,2-Diphenylhydrazine & $5 \times 10^{-5}$ & $4 \times 10^{-5}$ & & - \\
\hline Epichlorohydrin & $8 \times 10^{-3}$ & $4 \times 10^{-4}$ & - & - \\
\hline Formaldehyde (risk for $0.1 \mathrm{ppm}$ lifetime exp) & $2 \times 10^{-3}$ & $7 \times 10^{-4}$ & $3 \times 10^{-8}$ & - \\
\hline Heptachlor & $8 \times 10^{-6}$ & $8 \times 10^{-6}$ & - & - \\
\hline Heptachlor epoxide & $4 \times 10^{-6}$ & $6 \times 10^{-6}$ & - & - \\
\hline Hexachlorobenzene & $2 \times 10^{-5}$ & $2 \times 10^{-5}$ & - & - \\
\hline alpha-Hexachlorocyclohexane (alpha-HCH) & $6 \times 10^{-6}$ & $1 \times 10^{-5}$ & - & - \\
\hline beta-Hexachlorocyclohexane (beta-HCH) & $2 \times 10^{-5}$ & $2 \times 10^{-5}$ & - & - \\
\hline technical Hexachlorocyclohexane (t-HCH) & $2 \times 10^{-5}$ & $9 \times 10^{-6}$ & - & - \\
\hline Hexachloroethane & $2 \times 10^{-3}$ & $9 \times 10^{-4}$ & - & - \\
\hline Hydrazine/Hydrazine sulfate & $2 \times 10^{-6}$ & $2 \times 10^{-6}$ & - & - \\
\hline Nickel refinery dust & $4 \times 10^{-5}$ & - & $8 \times 10^{-6}$ & - \\
\hline Nickel subsulfide & $2 \times 10^{-5}$ & $2 \times 10^{-5}$ & - & - \\
\hline N-Nitroso-di-n-butylamine & $6 \times 10^{-6}$ & $3 \times 10^{-6}$ & - & - \\
\hline N-Nitrosodiethylamine & $2 \times 10^{-7}$ & $1 \times 10^{-6}$ & - & - \\
\hline N-Nitrosodimethylamine & $7 \times 10^{-7}$ & $2 \times 10^{-6}$ & - & - \\
\hline N-Nitrosopyrrolidine & $2 \times 10^{-5}$ & $2 \times 10^{-5}$ & - & - \\
\hline
\end{tabular}


Polychlorinated biphenyls (PCBs)

Propylene oxide

$1 \times 10^{-4} \quad 2 \times 10^{-5}$

$3 \times 10^{-3} \quad 3 \times 10^{-3}$

1,1,2,2-Tetrachloroethane

$2 \times 10^{-4} \quad 2 \times 10^{-4}$

Toxaphene

$3 \times 10^{-5} \quad 3 \times 10^{-5}$

1,1,2-Trichloroethane

2,4,6-Trichlorophenol

$6 \times 10^{-4} \quad 6 \times 10^{-4}$

Vinyl chloride

$3 \times 10^{-3}$

$1 \times 10^{-3 f}$

$5 \times 10^{-4}$

$1 \times 10^{-4}$

$4 \times 10^{-4}$

${ }^{a}$ RSCs at the $10^{-5}$ level. A smaller RSC indicates a higher estimated cancer potency. Red values indicate cancer potency estimates higher than EPA's. Green values indicate lower. ATSDR does not assess cancer potency. RSC $=10^{-5} /$ (unit risk).

b EPA RSCs are from the Integrated Risk Information System.

${ }^{c}$ California values are unit risks from the OEHHA Toxicity Criteria Database, converted to RSCs.

d Health Canada values are TC05s from the ITER database, converted to RSCs.

e Netherlands values are Cancer Risks from RIVM report 711701 025, expressed as RSCs.

${ }^{f}$ Most potent RSC of multiple listed values. 
TABLE S6. Oral Cancer Potency Assessment Comparison

Risk-specific doses $\left[\mathrm{mg} / \mathrm{kg}\right.$-day ${ }^{\mathrm{a}}$

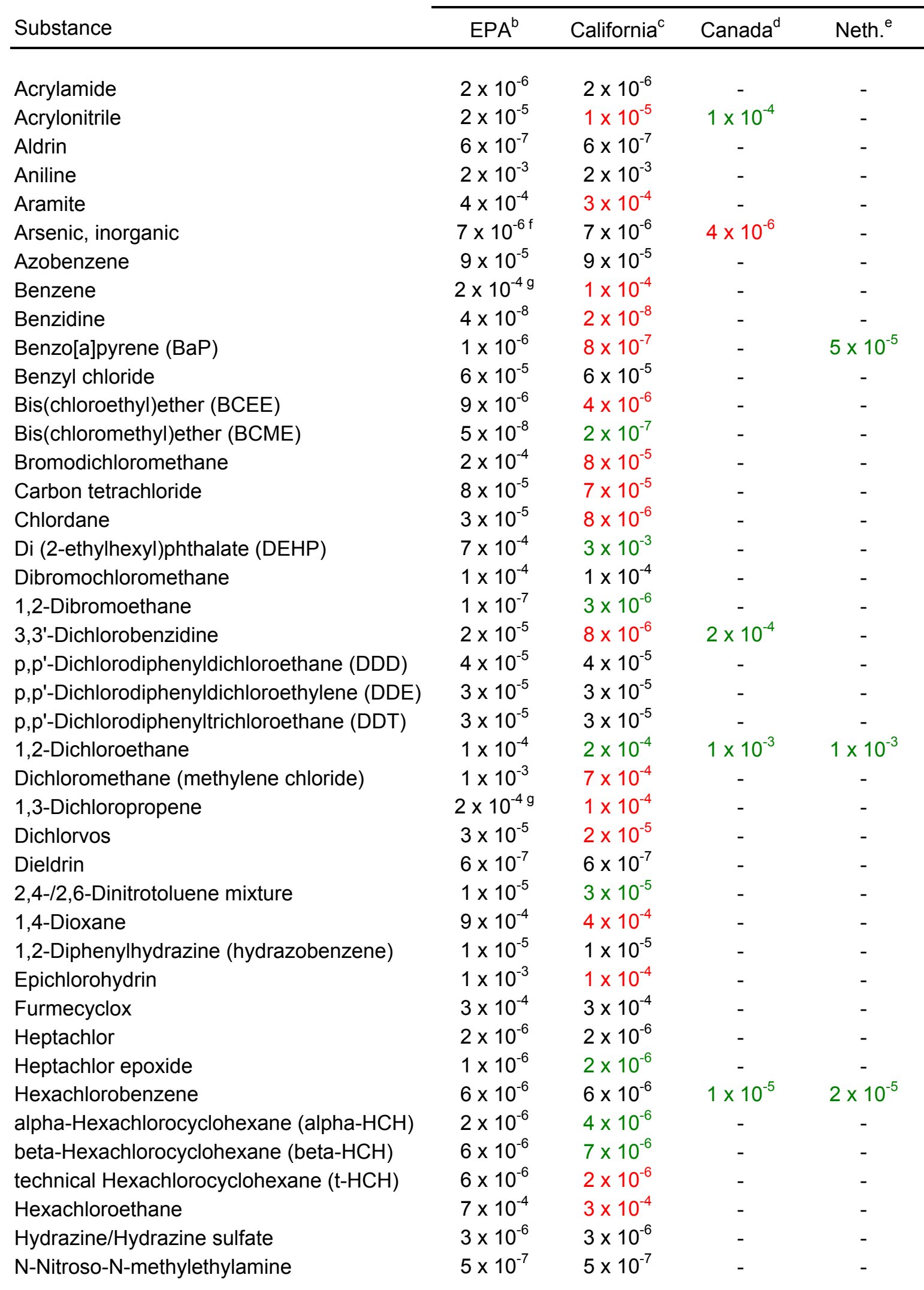


N-Nitroso-di-n-butylamine

$\mathrm{N}-\mathrm{Nitrosodi-N-propylamine}$

$\mathrm{N}-$ Nitrosodiethanolamine

$\mathrm{N}$-Nitrosodiethylamine

$\mathrm{N}$-Nitrosodimethylamine

$\mathrm{N}$-Nitrosodiphenylamine

$\mathrm{N}-$ Nitrosopyrrolidine

Pentachlorophenol

Polychlorinated biphenyls (PCBs)

Propylene oxide

1,1,2,2-Tetrachloroethane

Toxaphene

1,1,2-Trichloroethane

2,4,6-Trichlorophenol

Vinyl chloride

$\begin{array}{llcc}2 \times 10^{-6} & 9 \times 10^{-7} & - & - \\ 1 \times 10^{-6} & 1 \times 10^{-6} & - & - \\ 4 \times 10^{-6} & 4 \times 10^{-6} & - & - \\ 7 \times 10^{-8} & 3 \times 10^{-7} & - & - \\ 2 \times 10^{-7} & 6 \times 10^{-7} & 7 \times 10^{-6} & - \\ 2 \times 10^{-3} & 1 \times 10^{-3} & - & - \\ 5 \times 10^{-6} & 5 \times 10^{-6} & - & - \\ 8 \times 10^{-5} & 1 \times 10^{-4} & - & - \\ 5 \times 10^{-6} \mathrm{~g} & 2 \times 10^{-6} & - & - \\ 4 \times 10^{-5} & 4 \times 10^{-5} & - & - \\ 5 \times 10^{-5} & 4 \times 10^{-5} & - & - \\ 9 \times 10^{-6} & 8 \times 10^{-6} & - & - \\ 2 \times 10^{-4} & 1 \times 10^{-4} & - & - \\ 9 \times 10^{-4} & 1 \times 10^{-4} & - & - \\ 7 \times 10^{-6} \mathrm{~g} & 4 \times 10^{-5} & - & 6 \times 10^{-5}\end{array}$

${ }^{a}$ RSDs at the $10^{-5}$ level. A smaller RSD indicates a higher estimated cancer potency. Red values indicate cancer potency estimates higher than EPA's. Green values indicate lower. ATSDR does not assess cancer potency. RSD $=10^{-5} /$ (slope factor).

b EPA values are slope factors from the Integrated Risk Information System, converted to RSDs.

${ }^{c}$ California values are slope factors from the OEHHA Toxicity Criteria Database, converted to RSDs.

d Health Canada values are TD05's from the ITER database, converted to RSDs.

e Netherlands values are Cancer Risks from RIVM report 711701 025, expressed as RSDs.

${ }^{f}$ This IRIS estimate addresses skin cancer only and is not the basis for the current MCL for arsenic in drinking water.

${ }^{g}$ Most potent RSD of multiple listed values. 
FIGURE S3: Summary of Health Assessment Comparisons

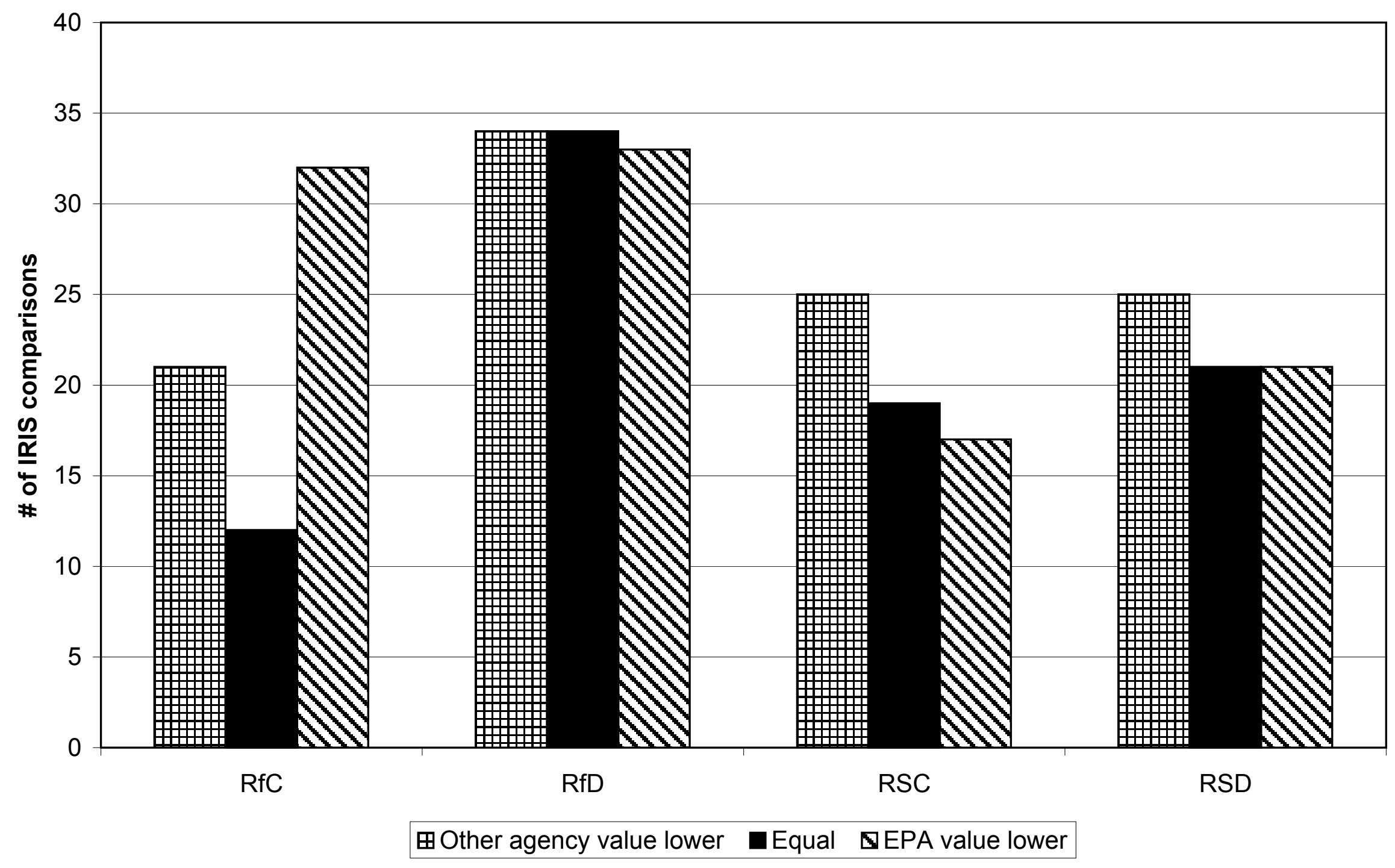




\title{
A Brief Survey of EPA Standard-Setting and Health Assessment
}

\author{
TIMOTHY C. BENNER
}

\section{Supporting Information}

\section{Additional Discussion on Cost-Benefit Analysis}

Cost-benefit analysis is one method for evaluating the need for and effectiveness of regulations. For ambient air quality standards and drinking water regulations, this involves comparing the implementation costs of reducing ambient levels of a pollutant with the monetized health benefits that result. This analysis may be performed either prospectively or retrospectively. As noted above, EPA may not consider implementation costs in setting the NAAQS (1) but must consider such costs in setting the NPDWR. However, Executive Order 12866 (58 F.R. 51735) requires agencies to perform prospective cost-benefit analyses for each significant regulatory action, regardless of whether these analyses may influence a particular policy decision. These analyses have often returned favorable results. For example, the regulatory impact analysis (RIA; 2) accompanying the most recent final NAAQS rule (62 F.R. 38651) predicted high net benefits for $\mathrm{PM}_{2.5}$ reductions. More recent rules have used higher estimates of $\mathrm{PM}_{2.5}$ benefits, based on more recent health studies, so this result would likely be even higher. The same RIA predicted net benefits for ozone reductions that may be either positive or negative. As with PM, more recent studies have suggested higher ozone health benefits (e.g., 3), so this result would also likely be more positive. Further, the RIA predicted results for a partial attainment strategy, rather than full attainment of the new NAAQS. It is worth noting that the National Research Council, in one review (4), generally approved of EPA's air pollution benefits analysis methodology, albeit with recommendations for improvement. For the NPDWR, the economic analysis accompanying the 1998 disinfectants and disinfection byproducts rule (63 F.R. 69390) predicted strong net benefits and speculated they might be even higher. The economic analysis for the 2001 arsenic rule (66 F.R. 6975) predicted somewhat higher costs than benefits, but a subsequent Science Advisory Board review (5) suggested potentially higher benefits and lower costs.

The Clean Air Act Amendments of 1990 (P.L. 101-549) require EPA to conduct cost-benefit analyses on the requirements of the Clean Air Act. These analyses are compiled and published as periodic reports, commonly known as "812 studies." The first report ( 6 ) was a retrospective analysis of 1970 to 1990 , which found that benefits exceeded costs by a large margin. The second report (7) was a prospective analysis of 1990 to 2010, and it also predicted that benefits would continue to significantly exceed costs. A third report is underway, extending the prospective analysis to 2020. Most of the monetized benefits in these analyses resulted from reductions in criteria pollutants, especially particulate matter, and further benefits of such reductions were identified but not quantified. It must be noted, however, that these analyses were not restricted to the NAAQS sections of the Clean Air Act. Costs and benefits included all the requirements of the Act and were not disaggregated by title or section. 


\section{Literature Cited}

(1) U.S. Supreme Court. Whitman v. American Trucking Assns., Inc., 531 U.S. 457, 2001.

(2) U.S. Environmental Protection Agency. Regulatory Impact Analyses for the Particulate Matter and Ozone National Ambient Air Quality Standards and Proposed Regional Haze Rule, 1997.

(3) Levy, J.I.; Carrothers, T.J.; Tuomisto, J.T.; Hammitt, J.K.; Evans, J.S. Environ. Health Perspect. 2001, 109, 9-20.

(4) National Research Council. Estimating the Public Health Benefits of Proposed Air Pollution Regulations; National Academies Press: Washington, DC, 2002.

(5) U.S. Environmental Protection Agency. Arsenic Rule Benefits Analysis: An SAB Review; EPA-SAB-EC-01-008, 2001.

(6) U.S. Environmental Protection Agency. The Benefits and Costs of the Clean Air Act, 1970 to 1990; EPA-410-R-97-002, 1997.

(7) U.S. Environmental Protection Agency. The Benefits and Costs of the Clean Air Act, 1990 to 2010: EPA Report to Congress; EPA-410-R-99-001, 1999. 